\title{
Fourvolutions and Automorphism Groups of Orbifold Lattice Vertex Operator Algebras
}

\author{
Hsian-Yang Chen* and Ching Hung Lam
}

\begin{abstract}
Let $L$ be an even positive definite lattice with no roots, i.e., $L(2)=\{x \in$ $L \mid(x \mid x)=2\}=\emptyset$. Let $g \in O(L)$ be an isometry of order 4 such that $g^{2}=-1$ on $L$. In this article, we determine the full automorphism group of the orbifold vertex operator algebra $V_{L}^{\widehat{g}}$. As our main result, we show that $\operatorname{Aut}\left(V_{L}^{\widehat{g}}\right)$ is isomorphic to $N_{\text {Aut }\left(V_{L}\right)}(\langle\widehat{g}\rangle) /\langle\widehat{g}\rangle$ unless $L \cong \sqrt{2} E_{8}$ or $B W_{16}$.
\end{abstract}

\section{Introduction}

Let $V$ be a vertex operator algebra (abbreviated as VOA) and let $g \in$ Aut $V$ be a finite automorphism of $V$. The fixed-point subalgebra

$$
V^{g}=\{v \in V \mid g v=v\}
$$

is often called an orbifold subVOA. Our main propose is to study the full automorphism group of the orbifold VOA $V^{g}$. It is clear that the normalizer $N_{\text {Aut }(V)}(\langle g\rangle)$ of $g$ stabilizes the orbifold VOA $V^{g}$ and it induces a group homomorphism $f: N_{\mathrm{Aut}(V)}(\langle g\rangle) /\langle g\rangle \rightarrow$ $\operatorname{Aut}\left(V^{g}\right)$. For generic cases, $\operatorname{Aut}\left(V^{g}\right)$ is often isomorphic to $N_{\operatorname{Aut}(V)}(\langle g\rangle) /\langle g\rangle$ but $\operatorname{Aut}\left(V^{g}\right)$ may be strictly bigger than $N_{\operatorname{Aut}(V)}(\langle g\rangle) /\langle g\rangle$. We call an automorphism $s \in \operatorname{Aut}\left(V^{g}\right)$ an extra automorphism if $s$ is not in the image of $f$, or equivalently, $s$ is not a restriction from an automorphism in $\operatorname{Aut}(V)$. When $V=V_{L}$ is a lattice VOA and $g$ is a lift of the $(-1)$ isometry of $L$, the full automorphism group of $V_{L}^{g}=V_{L}^{+}$has been determined in 22, 23. In particular, it was shown in 22,23 that the $\mathrm{VOA} V_{L}^{+}$contains an extra automorphism if and only if $L$ can be constructed by Construction B from a binary code (cf. Section 4.1). The main method is to study the orbit of $V_{L}^{-}=\left\{v \in V_{L} \mid g v=-v\right\}$ under the conjugate actions of $\operatorname{Aut}\left(V_{L}^{+}\right)$. Using a similar method, the automorphism groups of certain orbifold vertex operator algebras associated with some coinvariant lattice of the Leech lattice are also studied in [15, 17]. In addition, a sufficient condition for the existence of

Received March 11, 2021; Accepted April 29, 2021.

Communicated by Kunio Yamagata.

2020 Mathematics Subject Classification. 17B69.

Key words and phrases. lattice vertex operator algebra, automorphism group.

Chen is supported by MOST grant 109-2115-M-024-005-MY2 of Taiwan. Lam is supported by a research grant AS-IA-107-M02 of Academia Sinica and MOST grant 107-2115-M-001-003-MY3 of Taiwan.

*Corresponding author. 
extra automorphisms was discussed in 15,18 . The key idea is to generalize the triality automorphism defined in [11] (see also Section 4.1 and (4.1)) to other root lattices of type $A$. These results were also used in [3,4] to determine the automorphism groups of certain orbifold vertex operator algebras associated with the Leech lattice and to determine the full automorphism groups of certain holomorphic VOAs of central charge 24. One interesting question is if the sufficient condition mentioned in $[18$ is also a necessary condition for the existence of extra automorphisms? When $|g|=p$ is a prime and $L$ is rootless, it was shown in 18 that a cyclic orbifold $V_{L}^{\widehat{g}}$ contains extra automorphisms if and only if the rootless even lattice $L$ can be constructed by Construction B from a code over $\mathbb{Z}_{p}$ or is isometric to the coinvariant lattice of the Leech lattice associated with a certain isometry of order $p$.

In this article, we will continue our study of the full automrohism groups of certain cyclic orbifold of lattice vertex operator algebras and consider a case that $g \in O(L)$ has order 4 and $g^{2}=-1$ on $L$. Such an isometry is called a fourvolution in [13. As our main result, we will show that $\operatorname{Aut}\left(V_{L}^{\widehat{g}}\right)$ is isomorphic to $N_{\operatorname{Aut}\left(V_{L}\right)}(\langle\widehat{g}\rangle) /\langle\widehat{g}\rangle$ for any fourvolution $g \in O(L)$ unless $L \cong \sqrt{2} E_{8}$ or $B W_{16}$. When $L \cong \sqrt{2} E_{8}$ or $B W_{16}$, we also have $\operatorname{Aut}\left(V_{L}^{\widehat{g}}\right) \cong C_{\text {Aut }\left(V_{L}^{+}\right)}(\bar{g}) /\langle\bar{g}\rangle$, where $\bar{g}$ denotes the restriction of $\widehat{g}$ on $V_{L}^{+}$.

We shall note that for the cases studied in [3, 4, 15, 17, 18, $g$ often acts as an $n$-cycle on some root lattices of type $A_{n-1}$ and thus all $n$-th roots of unity (except 1) appear as eigenvalues of $g$, where $n=|g|$. Nevertheless, for a fourvolution $g \in O(L), g$ has only two eigenvalues $\sqrt{-1}$ and $-\sqrt{-1}$ and -1 is not an eigenvalue of $g$. Therefore, the condition discussed in [18] would never be satisfied when $g$ is a fourvolution. We hope that our study of fourvolutions and the automorphism groups of the corresponding orbifold VOA will provide some hints for the general cases.

\section{Preliminaries}

\subsection{Lattice VOAs and their automorphism groups}

We first recall the structure of the automorphism group of a lattice VOA $V_{L}$. Let $L$ be an even lattice with the (positive-definite) bilinear form $(\mid)$. We denote the group of isometries of $L$ by $O(L)$, i.e.,

$$
O(L)=\{g \in \mathrm{GL}(L) \mid(g x \mid g y)=(x \mid y) \text { for all } x, y \in L\}
$$

Let $\widehat{L}=\left\{ \pm e^{\alpha} \mid \alpha \in L\right\}$ be a central extension of $L$ by \pm 1 such that $e^{\alpha} e^{\beta}=(-1)^{(\alpha \mid \beta)} e^{\beta} e^{\alpha}$. Let $\operatorname{Aut}(\widehat{L})$ be the automorphism group of $\widehat{L}$ as a group. For $g \in \operatorname{Aut}(\widehat{L})$, let $\bar{g}$ be the map $L \rightarrow L$ defined by $g\left(e^{\alpha}\right) \in\left\{ \pm e^{\bar{g}(\alpha)}\right\}$. Let $O(\widehat{L})=\{g \in \operatorname{Aut}(\widehat{L}) \mid \bar{g} \in O(L)\}$. Then 
by [11, Proposition 5.4.1], we have an exact sequence

$$
1 \rightarrow \operatorname{Hom}(L, \mathbb{Z} / 2 \mathbb{Z}) \rightarrow O(\widehat{L}) \rightarrow O(L) \rightarrow 1 .
$$

It is known that $O(\widehat{L})$ is a subgroup of $\operatorname{Aut}\left(V_{L}\right)$ (cf. loc. cit.). Let

$$
N\left(V_{L}\right)=\left\langle\exp \left(a_{(0)}\right) \mid a \in\left(V_{L}\right)_{1}\right\rangle
$$

be the normal subgroup of $\operatorname{Aut}\left(V_{L}\right)$ generated by the inner automorphisms $\exp \left(a_{(0)}\right)$.

Theorem 2.1. [9] Let $L$ be a positive definite even lattice. Then

$$
\operatorname{Aut}\left(V_{L}\right)=N\left(V_{L}\right) O(\widehat{L}) .
$$

Moreover, the intersection $N\left(V_{L}\right) \cap O(\widehat{L})$ contains a subgroup $\operatorname{Hom}(L, \mathbb{Z} / 2 \mathbb{Z})$ and the quotient $\operatorname{Aut}\left(V_{L}\right) / N\left(V_{L}\right)$ is isomorphic to a quotient group of $O(L)$.

Remark 2.2. If $L(2)=\emptyset$, then $\left(V_{L}\right)_{1}=\operatorname{Span}\{\alpha(-1) \cdot 1 \mid \alpha \in L\}$. In this case, the normal subgroup $N\left(V_{L}\right)=\{\exp (\lambda \alpha(0)) \mid \alpha \in L, \lambda \in \mathbb{C}\}$ is abelian and we have $N\left(V_{L}\right) \cap O(\widehat{L})=$ $\operatorname{Hom}(L, \mathbb{Z} / 2 \mathbb{Z})$. Moreover, $\operatorname{Aut}\left(V_{L}\right) / N\left(V_{L}\right) \cong O(L)$ and we have an exact sequence

$$
1 \rightarrow N\left(V_{L}\right) \rightarrow \operatorname{Aut}\left(V_{L}\right) \stackrel{\varphi}{\rightarrow} O(L) \rightarrow 1
$$

Note also that $\exp \left(\lambda \alpha_{(0)}\right)$ acts trivially on $M(1)$ and $\exp (\lambda \alpha(0)) e^{\beta}=\exp (\lambda\langle\alpha, \beta\rangle) e^{\beta}$ for any $\lambda \in \mathbb{C}$ and $\alpha, \beta \in L$.

The following theorem can be proved by the same argument as in [19, Theorem 5.15].

Theorem 2.3. Let $L$ be a positive-definite rootless even lattice. Let $g$ be a fixed-point free isometry of $L$ of finite order and $\widehat{g}$ a lift of $g$ in $O(\widehat{L})$. Then we have the following exact sequences:

$$
\begin{gathered}
1 \longrightarrow \operatorname{Hom}\left(L /(1-g) L, \mathbb{C}^{*}\right) \longrightarrow N_{\mathrm{Aut}\left(V_{L}\right)}(\langle\widehat{g}\rangle) \stackrel{\varphi}{\longrightarrow} N_{O(L)}(\langle g\rangle) \longrightarrow 1, \\
1 \longrightarrow \operatorname{Hom}\left(L /(1-g) L, \mathbb{C}^{*}\right) \longrightarrow C_{\mathrm{Aut}\left(V_{L}\right)}(\widehat{g}) \stackrel{\varphi}{\longrightarrow} C_{O(L)}(g) \longrightarrow 1 .
\end{gathered}
$$

Let $h \in N_{\operatorname{Aut}\left(V_{L}\right)}(\langle\widehat{g}\rangle)$. Then it is clear that $h x \in V_{L}^{\widehat{g}}$ for any $x \in V_{L}^{\widehat{g}}$. Therefore, $N_{\text {Aut }\left(V_{L}\right)}(\langle\widehat{g}\rangle)$ acts on $V_{L}^{\widehat{g}}$ and there is a group homomorphism

$$
f: N_{\operatorname{Aut}\left(V_{L}\right)}(\langle\widehat{g}\rangle) /\langle\widehat{g}\rangle \longrightarrow \operatorname{Aut}\left(V_{L}^{\widehat{g}}\right) .
$$

The key question is to determine the image of $f$ and if $f$ is surjective. In particular, one would like to determine if there exist automorphisms in $\operatorname{Aut}\left(V_{L}^{\widehat{g}}\right)$ which are not induced from $N_{\operatorname{Aut}\left(V_{L}\right)}(\langle\widehat{g}\rangle)$. We call such an automorphism an extra automorphism.

Definition 2.4. An element $g \in O(L)$ is called a fourvolution if $g^{2}=-1$ on $L$. 
Lemma 2.5. [13] If $f$ is a fourvolution of a lattice $L$, then the adjoint of $1 \pm f$ is $1 \mp f$ and $1 \pm f$ is an isometry scaled by $\sqrt{2}$. Moreover, we have $L \geq(1-f) L \geq 2 L$ and $|L:(1-f) L|=|(1-f) L: 2 L|=|L / 2 L|^{1 / 2}$. In particular, $\operatorname{rank}(L)$ is even.

The main purpose of this article is to determine the full automorphism group of $V_{L}^{\widehat{g}}$ when $g$ is a fourvolution and $L(2)=\{x \in L \mid(x \mid x)=2\}=\emptyset$. In particular, we will show that $V_{L}^{\widehat{g}}$ contains no extra automorphisms unless $L \cong \sqrt{2} E_{8}$ or $B W_{16}$.

\section{Orbifolds of lattice VOAs having extra automorphisms}

Let $L$ be an even lattice with $L(2)=\emptyset$. Let $g \in O(L)$ be a fourvolution, i.e., $g^{2}=-1$. In this section, we will study $\operatorname{Aut}\left(V_{L}^{\widehat{g}}\right)$. In particular, we will determine when $\operatorname{Aut}\left(V_{L}^{\widehat{g}}\right)$ contains an extra automorphism.

First we recall that the orbifold VOA $V_{L}^{\widehat{g}}$ is $C_{2}$-cofinite and rational 5,21 and any irreducible $V_{L}^{\widehat{g}}$-module is a submodule of an irreducible $\widehat{g}^{i}$-twisted $V_{L}$-module for some $0 \leq i \leq|\widehat{g}|-1$ (see $[10])$.

For any irreducible (untwisted or twisted) module $M$ of $V_{L}, M$ is $\widehat{g}$-stable if $M \circ \widehat{g} \cong M$. In this case, $\widehat{g}$ acts on $M$ and we use

$$
M(j)=\left\{x \in M \mid \widehat{g} x=e^{2 \pi \sqrt{-1} j / n} x\right\}, \quad 0 \leq j \leq n-1, n=|\widehat{g}|
$$

to denote the eigenspaces of $\widehat{g}$ on $M$. Notice that $V_{\lambda+L}(j)$ is a simple current module of $V_{L}^{\widehat{g}}$ if $V_{\lambda+L}$ is $\widehat{g}$-stable, or equivalently, $(1-g) \lambda \in L$ (see for example [16]). By a result in [8], it is known that the number of inequivalent irreducible $\widehat{g}^{i}$-twisted modules is equal to the number of inequivalent irreducible $\widehat{g}^{i}$-stable modules of $V_{L}$. Moreover, all irreducible $\widehat{g}^{i}$-twisted modules are $\widehat{g}$-stable.

Let $\left(L^{*} / L\right)^{g^{i}}$ be the set of cosets of $L$ in $L^{*}$ fixed by $g^{i}$ and let $P_{0}^{g^{i}}: L^{*} \rightarrow \mathbb{Q} \otimes_{\mathbb{Z}} L^{g^{i}}$ be the orthogonal projection. Then $V_{L}$ has exactly $\left|\left(L^{*} / L\right)^{g^{i}}\right|$ irreducible $\widehat{g}^{i}$-twisted $V_{L}$-modules, up to isomorphism. The irreducible $\widehat{g}^{i}$-twisted $V_{L}$-modules have been constructed in 7,20 explicitly and are classified in [2]. They are given by

$$
V_{\lambda+L}\left[\widehat{g}^{i}\right]=M(1)\left[g^{i}\right] \otimes \mathbb{C}\left[P_{0}^{g^{i}}(\lambda+L)\right] \otimes T_{\widetilde{\lambda}} \quad \text { for } \lambda+L \in\left(L^{*} / L\right)^{g^{i}},
$$

where $M(1)\left[g^{i}\right]$ is the " $g^{i}$-twisted" free bosonic space, $\mathbb{C}\left[\lambda+P_{0}^{g^{i}}(L)\right]$ is a module for the group algebra of $P_{0}^{g^{i}}(L)$ and $T_{\widetilde{\lambda}}$ is an irreducible module for a certain " $g^{i}$-twisted" central extension of $L_{g^{i}}$ associated with $\lambda$ (see [20, Propositions 6.1 and 6.2] and 7, Remark 4.2] for detail).

Now assume that $V_{L}^{\widehat{g}}$ has an extra automorphism $\sigma$. Then by [24, Theorem 2.1] and $V_{L}(0) \circ \sigma=V_{L}^{\widehat{g}} \circ \sigma \cong V_{L}^{\widehat{g}}=V_{L}(0)$, we have

$$
\left\{V_{L}(r) \circ \sigma \mid 1 \leq r \leq 3\right\} \neq\left\{V_{L}(r) \mid 1 \leq r \leq 3\right\} .
$$


In other words, $V_{L}(1) \circ \sigma$ is isomorphic to a simple current module of $V_{L}^{\widehat{g}}$ not containing in $\left\{V_{L}(r) \mid 1 \leq r \leq 3\right\}$. By the classification of simple current irreducible $V_{L}^{\widehat{g}}$-modules, $V_{L}(1) \circ \sigma$ is either isomorphic to

(I) $V_{\lambda+L}(r)$ for some $\lambda \in L^{*} \backslash L$ with $(1-g) \lambda \in L$ and $0 \leq r \leq 3$;

(II) an irreducible $V_{L}^{\widehat{g}}$-submodule $V_{\lambda+L}^{T}\left[\widehat{g}^{2}\right](j)$ for some $0 \leq j \leq 3$; or

(III) an irreducible $V_{L}^{\widehat{g}}$-submodule $V_{\lambda+L}^{T}\left[\widehat{g}^{s}\right]_{\mathbb{Z}}$ with integral weights for some $s=1$ or 3 .

\subsection{Case (I): $\sigma$-conjugation of $V_{L}(1)$ is of untwisted type}

By the assumption, $V_{\lambda+L}(r)$ is a simple current module of $V_{L}^{\widehat{g}}$ and hence $(1-g) \lambda \in L 16$, Theorem 4.11]. Since $g^{2}=-1$ and there are only two eigenvalues $(\sqrt{-1}$ and $-\sqrt{-1})$ of $g$ on $\mathbb{C} \otimes_{\mathbb{Z}} L$, we have

$$
\operatorname{dim} V_{L}(1)_{1}=\operatorname{dim} V_{L}(3)_{1}=\frac{\operatorname{rank} L}{2} .
$$

Hence, we have $\operatorname{dim} V_{\lambda+L}(r)_{1}=\operatorname{rank} L / 2$, also. Moreover, we have $\operatorname{dim} V_{\lambda+L}(r)_{1}=\mid(\lambda+$ $L)(2) \mid / 4$ for any $0 \leq r \leq 3$ and thus

$$
|(\lambda+L)(2)|=2 \cdot \operatorname{rank} L
$$

Now set $N=\operatorname{Span}_{\mathbb{Z}}\{L, \lambda\}$.

Lemma 3.1. We have $|N / L|=2$.

Proof. First we note that $(1-g) \mu \in L$ for any $\mu \in \lambda+L$. In particular, $g \lambda \in \lambda+L$ and $(1-g) g \lambda=g \lambda-g^{2} \lambda=g \lambda+\lambda \in L \cap(2 \lambda+L)$. This implies $2 \lambda \in L$.

Lemma 3.2. The sublattice of $N$ spanned by $N(2)$ is isometric to the orthogonal sum of $n$ copies of $A_{1}$, where $n=\operatorname{rank} L$.

Proof. Let $\alpha \in(\lambda+L)(2)$. Then

$$
(\alpha \mid g \alpha)=\left(g \alpha \mid g^{2} \alpha\right)=-(g \alpha \mid \alpha)
$$

hence $(\alpha \mid g \alpha)=0$. Moreover, for any $\beta \in(\lambda+L)(2)$ and $\beta \notin\{ \pm \alpha, \pm g \alpha\}$, we have $(\alpha \mid \beta)=(g \alpha \mid \beta)=0$; otherwise, $(\alpha \mid \beta)= \pm 1$. This implies $\alpha+\beta$ or $\alpha-\beta$ are roots in $L$, which is impossible. Since $|(\lambda+L)(2)|=2 n, \lambda+L$ forms a semisimple root system of $A_{1}^{n}$ as desired.

Remark 3.3. By [22, Proposition 1.8], $L$ can be obtained by construction B from a binary code associated with an orthogonal basis in $(\lambda+L)(2)$. 
Let $\theta=\widehat{g}^{2}$. Then $\theta$ is a lift of the -1 isometry of $L$ and we use $V_{L}^{+}$to denote the fixed point $\operatorname{VOA} V_{L}^{\theta}$.

Proposition 3.4. Let $\sigma$ be an extra automorphism of $V_{L}^{\widehat{g}}$ such that $V_{L}(1) \circ \sigma \cong V_{\lambda+L}(r)$ for some $0 \leq r \leq 3$. Then $\sigma$ lifts to an automorphism of $V_{L}^{+}$.

Proof. Since $2 \lambda \in L$ and $V_{\lambda+L}(r)$ is a simple current module, $V_{\lambda+L}(r)^{\otimes 2}$ is isomorphic to an irreducible $V_{L}^{\widehat{g}}$-submodule of $V_{L}$. Moreover, $V_{L}(1)^{\otimes 2} \cong V_{L}(2)$ has top weight $>1$.

Since $V_{L}(2)$ is the only irreducible $V_{L}^{\widehat{g}}$-submodule of $V_{L}$ which has the top weight $>1$, we have $V_{\lambda+L}(r)^{\bigotimes 2}=\left(V_{L}(1) \circ \sigma\right)^{\bigotimes 2} \cong V_{L}(2)$. Therefore, $\sigma$ preserves the subspace $V_{L}^{+}=V_{L}^{\widehat{g}}+V_{L}(2)$ and $\sigma$ lifts to an automorphism of $V_{L}^{+}$by [24, Theorem 2.1].

3.2. Case (II): $\sigma$-conjugation of $V_{L}(1)$ is contained in $\widehat{g}^{2}$-twisted module Next, we consider Case (II), i.e., $V_{L}(1) \circ \sigma \cong V_{\lambda+L}^{T}\left[\widehat{g}^{2}\right](j)$ for some $0 \leq j \leq 3$.

In this case, $V_{\lambda+L}^{T}\left[\widehat{g}^{2}\right]_{1} \neq 0$. Since $\widehat{g}^{2}$ is a lift of the -1 isometry, it is proved in 22$]$ that $n=\operatorname{rank} L=8$ or 16 . Moreover, $L^{*} / L$ is an elementary abelian 2-group and $L \cong \sqrt{2} E_{8}$ if $n=8$ and $L \cong B W_{16}$ if $n=16$.

3.3. Case (III): $\sigma$-conjugation of $V_{L}(1)$ is contained in $\widehat{g}$-twisted $V_{L}$-module Next, we consider Case (III), i.e., $V_{L}(1) \circ \sigma \cong V_{\lambda+L}^{T}\left[\widehat{g}^{s}\right]_{\mathbb{Z}}$ for some $s=1$ or 3 .

Since $g$ is fixed point free on $L$, the irreducible $\widehat{g}^{s}$-twisted module $V_{\lambda+L}^{T}\left[\widehat{g}^{s}\right]$, for $s=1$ or 3 , is given by

$$
V_{\lambda+L}^{T}\left[\widehat{g}^{s}\right]=M(1)\left[g^{s}\right] \otimes T_{\widetilde{\lambda}} .
$$

For a fourvolution $g$ and $s$ odd, the conformal weight of $V_{\lambda+L}^{T}\left[\widehat{g}^{s}\right]$ (see $[7,20]$ ) is given by

$$
\varepsilon=\frac{3 n}{4 \times 4^{2}} .
$$

That $V_{L}(1) \circ \sigma \cong V_{\lambda+L}^{T}\left[\widehat{g}^{s}\right]_{\mathbb{Z}}$ implies $\varepsilon \leq 1$ and $\varepsilon \in \frac{1}{4} \mathbb{Z}$. It is easy to verify that $n=16$ is the only solution and $\varepsilon=3 / 4$. In this case, $V_{\lambda+L}^{T}\left[\widehat{g}^{s}\right]_{\mathbb{Z}}$ is a simple current module for $V_{L}^{\widehat{g}}$; hence we have $(1-g) L^{*} \leq L$ and $\operatorname{dim} T_{\widetilde{\lambda}}=\left[L:(1-g) L^{*}\right]^{1 / 2}$ 1, Corollary 3.7]. Since $\varepsilon=3 / 4$ and $\operatorname{dim}\left(V_{\lambda+L}^{T}\left[\widehat{g}^{s}\right]\right)_{1}=\operatorname{dim} V_{L}(1)_{1}=\operatorname{rank} L / 2$, we have $\operatorname{dim} T_{\widetilde{\lambda}}=1$ and $L=(1-g) L^{*}$. Moreover,

$$
\left|L^{*} / L\right|=|L /(1-g) L|=|\operatorname{det}(1-g)|=2^{n / 2} .
$$

For $n=16$, we have $\left|L^{*} / L\right|=2^{8}$. Note that $L_{2}=\emptyset$ and there is only one such lattice up to isometry and $L \cong B W_{16}[22$, Proposition 1.9].

By our assumption, $V_{L}(1) \circ \sigma \cong V_{\lambda+L}^{T}\left[\widehat{g}^{s}\right]_{\mathbb{Z}}$ for some $\lambda \in L^{*}$ and $s=1$ or 3 and we have

$$
\left(V_{\lambda+L}^{T}\left[\widehat{g}^{s}\right]_{\mathbb{Z}}\right)^{\bigotimes 2} \cong V_{L}(2) \circ \sigma .
$$


On the other hand, $\left(V_{\lambda+L}^{T}\left[\widehat{g}^{s}\right]_{\mathbb{Z}}\right)^{\bigotimes 2}$ is isomorphic to an irreducible $V_{L}^{\widehat{g}}$-submodule of a $\widehat{g}^{2}$ twisted module $V_{\mu+L}^{T}\left[\widehat{g}^{2}\right]$ for some $\mu \in L^{*}$.

When $L \cong B W_{16}$, the conformal weight of $V_{\mu+L}^{T}\left[\widehat{g}^{2}\right]$ is 1 for any $\mu \in L^{*}$ and $V_{\mu+L}^{T}\left[\widehat{g}^{2}\right](j)$, $0 \leq j \leq 3$, has conformal weight either 1 or $3 / 2$. It is a contradiction since the conformal weight of $V_{L}(2)$ is $\geq 2$. That means Case (III) does not occur.

\section{Automorphism groups of $V_{L}^{\widehat{g}}$}

In this section, we will study the automorphism groups of $V_{L}^{\widehat{g}}$ when $g$ is a fourvolution, i.e., $g^{2}=-1$. Let $\theta=\widehat{g}^{2}$. Then $\theta$ is a lift of the -1 isometry of $L$ and we use $V_{L}^{+}$to denote the fixed point $\operatorname{VOA} V_{L}^{\theta}$.

\section{1. $L \not \sqrt{2} E_{8}$ nor $B W_{16}$}

First we assume that $L \nsubseteq \sqrt{2} E_{8}$ nor $B W_{16}$.

Theorem 4.1. Let $L$ be even lattice with $L(2)=\emptyset$ and $g \in O(L)$ a fourvolution. Suppose $L \nsubseteq \sqrt{2} E_{8}$ nor $B W_{16}$. Then $\operatorname{Aut}\left(V_{L}^{\widehat{g}}\right) \cong C_{\text {Aut }\left(V_{L}^{+}\right)}(\bar{g}) /\langle\bar{g}\rangle$, where $\bar{g}$ denotes the restriction of $\widehat{g}$ on $V_{L}^{+} \cong V_{L}^{\widehat{g}^{2}}$.

Proof. Suppose $L \nsubseteq \sqrt{2} E_{8}$ nor $B W_{16}$. Then either $V_{L}^{\widehat{g}}$ has no extra automorphisms or Case (I) holds. In either cases, automorphisms of $V_{L}^{\widehat{g}}$ will stabilize $V_{L}^{\widehat{g}^{2}}=V_{L}(0) \oplus V_{L}(2)$ by Proposition 3.4. Since $V_{L}^{\widehat{g}^{2}}$ is a simple current extension of $V_{L}^{\widehat{g}}$, the result then follows from [22, Theorem 3.3].

Recall that $g^{2}=-1$ and hence $\widehat{g}^{2}$ is a lift the $(-1)$-isometry. The automorphism group for the fixed point VOA $V_{L}^{+}$has been determined in 22 . We will recall some results in 22$]$. First, we recall the Construction $\mathrm{B}$ of a lattice from a binary code $C$.

Let $C$ be a doubly even binary code of length $n$ and let $\mathcal{B}=\left\{\alpha_{i} \mid i \in\{1, \ldots, n\}\right\}$ be an orthogonal basis of $\mathbb{R}^{n}$ of norm 2 , i.e., $\left\langle\alpha_{i}, \alpha_{j}\right\rangle=2 \delta_{i, j}$. For $c=\left(c_{1}, \ldots, c_{n}\right) \in \mathbb{Z}_{2}^{n}$, set

$$
\alpha_{c}=\sum_{i=1}^{n} c_{i} \alpha_{i}
$$

The lattice

$$
L_{B}(C)=\sum_{c \in C} \mathbb{Z} \frac{1}{2} \alpha_{c}+\sum_{i, j \in\{1, \ldots, n\}} \mathbb{Z}\left(\alpha_{i}+\alpha_{j}\right)
$$

is often referred as to the lattice obtained by Construction B from $C$ associated with $\mathcal{B}$. Note that $L_{B}(C)$ always contains the sublattice $\sum_{i, j \in\{1, \ldots, n\}} \mathbb{Z}\left(\alpha_{i}+\alpha_{j}\right) \cong \sqrt{2} D_{n}$. 
Set $L_{A}(C)=L_{B}(C)+\mathbb{Z} \alpha_{1}$. Then $\mathcal{B}=\left\{\alpha_{i} \mid i=1, \ldots, n\right\}<L_{A}(C)$. Now fix $a_{k} \in \widehat{L_{A}}(C)$ for each $k \in\{1, \ldots, n\}$ such that $\bar{a}_{k}=\alpha_{k}$ and define

$$
\sigma=\prod_{k=1}^{n} \exp \left((1+\sqrt{-2})\left(a_{k}\right)_{0}\right) \exp \left(\sqrt{-\frac{1}{2}}\left(a_{k}^{-1}\right)_{0}\right) \exp \left((-1+\sqrt{-2})\left(a_{k}\right)_{0}\right) .
$$

Then $\sigma$ is an automorphism of the $\operatorname{VOA} V_{L_{A}(C)}$. Indeed, $\sigma$ defines an automorphism of $V_{L_{B}(C)}^{+}$(cf. 11,22$\left.]\right)$.

Remark 4.2. We shall note that there are usually several choices for the orthogonal basis $\mathcal{B}$ and the automorphism $\sigma$ depends on the choice of the orthogonal basis $\mathcal{B}=\left\{\alpha_{1}, \ldots, \alpha_{n}\right\}$. Moreover, for each $\alpha_{k} \in \mathcal{B}$,

$$
\sigma: \quad \alpha_{k}(-1) \mapsto e^{\alpha_{k}}+e^{-\alpha_{k}}, \quad e^{\alpha_{k}}+e^{-\alpha_{k}} \mapsto \alpha_{k}(-1), \quad e^{\alpha_{k}}-e^{-\alpha_{k}} \mapsto-\left(e^{\alpha_{k}}-e^{-\alpha_{k}}\right) .
$$

The following theorem can be found in 22$]$.

Theorem 4.3. [22, Proposition 3.16] Let $L$ be an even lattice such that $L(2)=\emptyset$. Then $\operatorname{Aut}\left(V_{L}^{+}\right)$is generated by $O(\widehat{L}) /\langle\theta\rangle$ and the extra automorphisms defined as in (4.1). In particular, $\operatorname{Aut}\left(V_{L}^{+}\right)$contains an extra automorphism if and only if $L$ can be constructed by Construction $B$ from some binary code $C$.

Theorem 4.4. Suppose $L(2)=\emptyset$ and $L \nsubseteq \sqrt{2} E_{8}$ nor $B W_{16}$. Then for a fourvolution $g \in O(L)$, we have

$$
\operatorname{Aut}\left(V_{L}^{\widehat{g}}\right) \cong N_{\operatorname{Aut}\left(V_{L}\right)}(\langle\widehat{g}\rangle) /\langle\widehat{g}\rangle
$$

Proof. Suppose false. Then there is $\sigma \in \operatorname{Aut}\left(V_{L}^{\widehat{g}}\right)$ such that

$$
\left\{V_{L}(i) \circ \sigma \mid 0 \leq i \leq 3\right\} \neq\left\{V_{L}(i) \mid 0 \leq i \leq 3\right\}
$$

Since $L \nsucceq \sqrt{2} E_{8}$ nor $B W_{16}$, only Case (I) can occur; hence, there exist $\lambda \in L^{*}$ and $0 \leq j \leq 3$ such that $V_{L}(1) \circ \sigma \cong V_{\lambda+L}(j)$.

Recall from the discussion in Case (I), we have $|(\lambda+L)(2)|=2 n$ and $(\lambda+L)(2)$ forms a root system of type $A_{1}^{n}$. Moreover, $\sigma$ lifts to an automorphism of $V_{L}^{+}=V_{L}(0) \oplus V_{L}(2)$. In this case, $V_{L}^{-}=V_{L}(1) \oplus V_{L}(3)$ and $V_{L}^{-} \circ \sigma \cong V_{\lambda+L}^{\varepsilon}$ for some $\varepsilon= \pm 1$. Moreover, $N=\operatorname{Span}_{Z}\{L, \lambda\}=L_{A}(C)$ for some binary code $C$ and $L=L_{B}(C)$.

Fix an orthogonal basis $\mathcal{B}_{\lambda}<(\lambda+L)(2)$, i.e., a set of simple roots for $(\lambda+L)(2)$, and let $\sigma_{\lambda}$ be the extra automorphism associated with the basis $\mathcal{B}_{\lambda}$ as defined above. Then $\sigma \sigma_{\lambda}^{-1}$ stabilize $V_{L}^{-}$and hence $\sigma \sigma_{\lambda}^{-1} \in O(\widehat{L}) 22$ and $\sigma=h \sigma_{\lambda}$ for some $h \in O(\widehat{L})$.

Now let $\alpha_{1}, \alpha_{2} \in \mathcal{B}_{\lambda}$ such that $g$ maps $\alpha_{1} \rightarrow \alpha_{2} \rightarrow-\alpha_{1} \rightarrow-\alpha_{2}$. Then $\widehat{g}$ fixes the element $\left(e^{\alpha_{1}}+e^{-\alpha_{1}}\right)_{-1}\left(e^{\alpha_{2}}+e^{-\alpha_{2}}\right)$. Note that $\left(e^{\alpha_{1}}+e^{-\alpha_{1}}\right),\left(e^{\alpha_{2}}+e^{-\alpha_{2}}\right) \in V_{\lambda+L}$ and hence $\left(e^{\alpha_{1}}+e^{-\alpha_{1}}\right)_{-1}\left(e^{\alpha_{2}}+e^{-\alpha_{2}}\right) \in V_{2 \lambda+L}=V_{L}$. However, for any $h \in O(\widehat{L})$,

$$
h \sigma_{\lambda}\left(\left(e^{\alpha_{1}}+e^{-\alpha_{1}}\right)_{-1}\left(e^{\alpha_{2}}+e^{-\alpha_{2}}\right)\right)=h\left(\alpha_{1}(-1) \alpha_{2}(-1) \cdot 1\right)=\bar{h} \alpha_{1}(-1) \bar{h} \alpha_{2}(-1) \cdot 1,
$$

which is not fixed by $\widehat{g}$. It contradicts that $h \sigma_{\lambda}=\sigma \in \operatorname{Aut}\left(V_{L}^{\widehat{g}}\right)$. 


\subsection{Case: $L \cong \sqrt{2} E_{8}$ or $B W_{16}$}

Next we consider the case $L=\sqrt{2} E_{8}$ or $B W_{16}$. In both cases, $L^{*} / L$ is an elementary abelian 2-group of order $2^{8}$. It is also known [22] that the set of inequivalent irreducible modules $\operatorname{Irr}\left(V_{L}^{+}\right)$of $V_{L}^{+}$forms an elementary abelian 2-group under the fusion rules 22. Recall that $V_{\lambda+L}^{T}[\theta]_{\mathbb{Z}}$ has the conformal weight 1 if $L \cong \sqrt{2} E_{8}$ or $B W_{16}$. Moreover, $\operatorname{dim}\left(V_{\lambda+L}^{T}[\theta]_{1}\right)=8$ if $L \cong \sqrt{2} E_{8}$ and $\operatorname{dim}\left(V_{\lambda+L}^{T}[\theta]_{1}\right)=16$ if $L \cong B W_{16}$.

Lemma 4.5. Let $L=\sqrt{2} E_{8}$ or $B W_{16}$. Suppose there is a $\sigma \in V_{L}^{\widehat{g}}$ such that $V_{L}(1) \circ \sigma \cong$ $V_{\lambda+L}^{T}\left[\widehat{g}^{2}\right](j)$. Then $\sigma$ stabilizes $V_{L}^{+}=V_{L}(0) \oplus V_{L}(2)$ by the conjugate action.

Proof. For $L \cong \sqrt{2} E_{8}$ or $B W_{16}$, we have $V_{\lambda+L}^{T}\left[\widehat{g}^{2}\right]_{\mathbb{Z}} \otimes_{V_{L}^{+}} V_{\lambda+L}^{T}\left[\widehat{g}^{2}\right]_{\mathbb{Z}}=V_{L}^{+}$and hence $V_{\lambda+L}^{T}\left[\widehat{g}^{2}\right](j)^{\bigotimes 2}<V_{L}^{+}$. Therefore, $V_{\lambda+L}^{T}\left[\widehat{g}^{2}\right](j)^{\bowtie 2} \cong V_{L}(2)$ as $V_{L}(2)$ is the only irreducible $V_{L}^{\widehat{g}}$ module with the top weight $>1$ in $V_{L}^{+}$.

As a consequence, we have

Proposition 4.6. Let $L \cong \sqrt{2} E_{8}$ or $B W_{16}$ and $g \in O(L)$ a fourvolution. Then,

$$
\operatorname{Aut}\left(V_{L}^{\widehat{g}}\right) \cong C_{\operatorname{Aut}\left(V_{L}^{+}\right)}(\bar{g}) /\langle\bar{g}\rangle,
$$

where $\bar{g}$ denotes the restriction of $\widehat{g}$ on $V_{L}^{+} \cong V_{L}^{\widehat{g}^{2}}$.

$$
\text { 4.2.1. } L \cong \sqrt{2} E_{8}
$$

In this case, the isometry group $O(L)$ is the Weyl group of $E_{8}$ and it has the shape $2 . \Omega_{8}^{+}(2) .2$. A fourvolution $g$ corresponds to a $2 C$-element in $\Omega_{8}^{+}(2)$. An irreducible $\widehat{g}^{2}$ twisted module $V_{\lambda+L}^{T}\left[\widehat{g}^{2}\right]$ has the top weight $1 / 2$ and $\operatorname{dim}\left(V_{\lambda+L}^{T}\left[\widehat{g}^{2}\right]\right)_{1}=8$.

Recall that the set of inequivalent irreducible modules for $V_{L}^{+}$forms an elementary abelian group of order $2^{10}$ under the fusion rules and the automorphism group $V_{\sqrt{2} E_{8}}^{+} \cong$ $G O_{10}^{+}(2)$ (cf. 12,22$]$ ).

Theorem 4.7. Let $L \cong \sqrt{2} E_{8}$ and $g \in O\left(\sqrt{2} E_{8}\right)$ a fourvolution. Then $\operatorname{Aut}\left(V_{L}^{\widehat{g}}\right)$ has order $2^{18} \cdot 3^{2} \cdot 5$ and has the shape $\left[2^{14}\right]$. Sym $_{6}$.

Proof. We first recall that $\operatorname{dim}\left(V_{\sqrt{2} E_{8}}^{+}\right)_{2}=156$ and $\left(V_{\sqrt{2} E_{8}}^{+}\right)_{2}$ decomposed as $1+155$ as a sum of irreducible representations of $G_{10}^{+}(2)$. By a direct calculation, it is easy to show that $\operatorname{dim}\left(V_{\sqrt{2} E_{8}}^{\widehat{g}}\right)_{2}=76$. Therefore, $\bar{g}$ acts on $\left(V_{\sqrt{2} E_{8}}^{+}\right)_{2}$ with trace -4 , i.e., $\bar{g}$ corresponds to a $2 C$ element of $G O_{10}^{+}(2)$ using the Atlas notation. In this case, the centralizer $C_{G O_{10}^{+}(2)}(\bar{g})$ has order $2^{19} \cdot 3^{2} \cdot 5$ and has the shape $\left[2^{15}\right] . S y m_{6}$. By Proposition 4.6 , we have the desired result. 


$$
\text { 4.2.2. } L \cong B W_{16}
$$

In this case, $L^{*} / L \cong 2^{8}$ and $\left|L / 2 L^{*}\right|=2^{8}$.

First we recall few facts about the isometry group $O(L)$. It is clear that $O(L)$ acts naturally on the discriminant group $L^{*} / L \cong 2^{8}$ and it induces a group homomorphism $s: O(L) \rightarrow O\left(L^{*} / L, q\right) \cong G O_{8}^{+}(2)$.

Let $K=\operatorname{ker} s$. Then $K$ is isomorphic to an extra special group $2^{1+8}$ and $\operatorname{Im} s \cong \Omega_{8}^{+}(2)$. Therefore, $O(L) \cong 2^{1+8} . \Omega_{8}^{+}(2)$ (see $[6,13]$ ). A fourvolution $g$ can be decomposed into the product of two involutions $t_{M}, t_{N}$ associated with two $\sqrt{2} E_{8}$ sublattices $M$ and $N$ such that $L=M+N$. Recall that $t_{M}$ is an isometry of $L$ which acts as -1 on $M$ and acts as 1 on the annihilator $\operatorname{Ann}_{L}(M)=\{x \in L \mid(x, y)=0$ for all $y \in M\}$. It is well-defined since $2 M^{*}<M$ (cf. 13, 14]). These two involutions $t_{M}, t_{N}$ generate a dihedral group of order 8 and is contained in the extra-special 2-group $2^{1+8}$. In particular, $\left\langle t_{M}, t_{N}\right\rangle$ acts trivially on the discriminant group $L^{*} / L\left(\cong 2^{8}\right)$. Recall also from 13 that the quotient group $K . C_{O(L)}\left(t_{M}\right) / K$ of the centralizer of $t_{M}$ determines a maximal parabolic subgroup of the shape $2^{6} . \Omega_{6}^{+}(2)$ in $\Omega_{8}^{+}(2)$, which corresponds to the stabilizer of a singular vector $u_{M}$. Similarly, $K . C_{O(L)}\left(t_{N}\right) / K \cong 2^{6} . \Omega_{6}^{+}(2)$ corresponds to the stabilizer of another singular vector $u_{N}$. Note that $g=t_{M} t_{N} \in K$ and we have $N_{O(L)}(\langle g\rangle) / K \cong S p_{6}(2)$, which is the stabilizer of a non-singular vector $u_{M}+u_{N}$. Indeed, $N_{O(L)}(\langle g\rangle) \cong 2^{1+8} \cdot S p_{6}(2)$. By Theorem 2.3, we also have the following lemma.

Lemma 4.8. Let $L=B W_{16}$ and $g \in O(L)$ a fourvolution. Then,

$$
N_{\text {Aut }\left(V_{L}\right)}(\langle\widehat{g}\rangle) /\langle\widehat{g}\rangle \cong 2^{8} \cdot\left(2^{7} \cdot S p_{6}(2)\right) \text {. }
$$

Remark 4.9. We note that the Barnes-Wall lattice $B W_{16}$ can also be realized as the coinvariant lattice of the Leech lattice associated with a $4 A$ element of the Conway group $\mathrm{Co}_{0}$.

Next we review the irreducible modules for $V_{B W_{16}}^{\widehat{g}}$ for a fourvolution $g \in O\left(B W_{16}\right)$. Recall that there are two types of irreducible modules for $V_{B W_{16}}^{\widehat{g}}$ :

Untwisted type: $V_{\lambda+L}(j), \lambda \in L^{*}$ and $j=0,1,2,3$; and

Twisted type: $V_{\lambda+L}^{T}\left[\widehat{g}^{i}\right](j), \lambda \in L^{*}, i=1,2,3$ and $j=0,1,2,3$.

Set $F=\left\{V_{L}^{T}\left[\widehat{g}^{i}\right](j) \mid i, j=0,1,2,3\right\}$ and $E=\left\{V_{\lambda+L}(0) \mid \lambda+L \in L^{*} / L\right\}$, where $V_{L}^{T}\left[\widehat{g}^{0}\right](j)=V_{L}(j)$.

For $L=B W_{16}$, we have $(1-g) L^{*}=L$ for any fourvolution $g$. By [16], all irreducible modules of $V_{L}^{\widehat{g}}$ are simple current modules. Moreover, the set of inequivalent irreducible modules $\operatorname{Irr}\left(V_{B W_{16}}^{\widehat{g}}\right)$ of $V_{B W_{16}}^{\widehat{g}}$ forms an abelian group under the fusion rules and it has a quadratic form $q$ defined by conformal weights modulo $\mathbb{Z}$. By the discussion in [16], we 
also know that $E \cong 2^{8}, F \cong 4^{2}$ as abelian groups and $\operatorname{Irr}\left(V_{B W_{16}}^{\widehat{g}}\right)=E \times F$. Note also that $F$ is the subgroup generated by $V_{L}(1)$ and $V_{L}^{T}[g](0)$.

Theorem 4.10. Let $L \cong B W_{16}$ and $g \in O\left(B W_{16}\right)$ a fourvolution. Then $\operatorname{Aut}\left(V_{L}^{\widehat{g}}\right) \cong$ $\left[2^{15}\right] .\left(S p_{6}(2) \times 2\right)$.

Proof. By the same argument as in the proof of Theorem 4.4, there is no automorphisms $s \in \operatorname{Aut}\left(V_{L}^{\widehat{g}}\right)$ such that $V_{L}(1) \circ s \cong V_{\lambda+L}(j)$ for any $\lambda \in L^{*} \backslash L$ and $0 \leq j \leq 3$. In other words, if $s$ stabilizes the set of untwisted irreducible modules, then it also stabilizes the set $\left\{V_{L}(i) \mid i=0,1,2,3\right\}$ and is thus contained in

$$
S=\operatorname{Stab}_{\operatorname{Aut}\left(V_{B W_{16}}^{\widehat{g}}\right)}\left(\left\{V_{L}(i) \mid i=0,1,2,3\right\}\right) \cong N_{\mathrm{Aut}\left(V_{L}\right)}(\langle\widehat{g}\rangle) /\langle\widehat{g}\rangle
$$

An extra automorphism $s$ thus maps $V_{L}(1)$ to a twisted type module. Note that $L^{*} / L \cong$ $L /(1-g) L$ and for any $\lambda+L \in L^{*} / L$, there is an $f \in \operatorname{Hom}\left(L /(1-g) L, \mathbb{C}^{*}\right)$ such that $V_{\lambda+L}^{T}[\widehat{g}](0) \cong V_{L}^{T}[\widehat{g}](0) \circ f$. Without loss of generality, we may assume $V_{L}(1) \circ s \cong V_{L}^{T}[\widehat{g}](0)$ or $V_{L}^{T}\left[\widehat{g}^{3}\right](0)$. In this case, $s$ stabilizes $F$. Note that the orthogonal group $O(F, q) \cong 2^{2}$. Moreover, there is an automorphism $\sigma \in \operatorname{Aut}\left(V_{B W_{16}}^{\widehat{g}}\right)$ such that $V_{L}(1) \circ \sigma \cong V_{L}^{T}[\widehat{g}](0)$ by Remark 4.9 and [17, Theorem 3.4] and we have the desired result.

\section{References}

[1] T. Abe, C. H. Lam and H. Yamada, Extensions of tensor products of $\mathbb{Z}_{p}$-orbifold models of the lattice vertex operator algebra $V_{\sqrt{2} A_{p-1}}$, J. Algebra 510 (2018), 24-51.

[2] B. Bakalov and V. G. Kac, Twisted modules over lattice vertex algebras, in: Lie Theory and its Applications in Physics V, 3-26, World Sci. Publ., River Edge, NJ, 2004.

[3] K. Betsumiya, C. H. Lam and H. Shimakura, Automorphism group of orbifold vertex operator algebras associated with the Leech lattice: Non-prime cases, in preparation.

[4] _ Holomorphic vertex operator algebras of central charge 24 and their automorphism groups, in preparation.

[5] S. Carnahan and M. Miyamoto, Regularity of fixed-point vertex operator subalgebras, arXiv:1603.05645.

[6] J. H. Conway and N. J. A. Sloane, Sphere Packings, Lattices and Groups, Third edition, Grundlehren der Mathematischen Wissenschaften 290, Springer-Verlag, New York, 1999. 
[7] C. Dong and J. Lepowsky, The algebraic structure of relative twisted vertex operators, J. Pure Appl. Algebra 110 (1996), no. 3, 259-295.

[8] C. Dong, H. Li and G. Mason, Modular-invariance of trace functions in orbifold theory and generalized Moonshine, Comm. Math. Phys. 214 (2000), no. 1, 1-56.

[9] C. Dong and K. Nagatomo, Automorphism groups and twisted modules for lattice vertex operator algebras, in: Recent Developments in Quantum Affine Algebras and Related Topics (Raleigh, NC, 1998), 117-133, Contemp. Math. 248, Amer. Math. Soc., Providence, RI, 1999.

[10] C. Dong, L. Ren and F. Xu, On orbifold theory, Adv. Math. 321 (2017), 1-30.

[11] I. Frenkel, J. Lepowsky and A. Meurman, Vertex operator algebras and the Monster, Pure and Applied Mathematics 134, Academic Press, Boston, MA, 1988.

[12] R. L. Griess, Jr., A vertex operator algebra related to $E_{8}$ with automorphism group $\mathrm{O}^{+}(10,2)$, in: The Monster and Lie Algebras (Columbus, OH, 1996), 43-58, Ohio State Univ. Math. Res. Inst. Publ. 7, de Gruyter, Berlin, 1998.

[13] _ Pieces of $2^{d}$ : Existence and uniqueness for Barnes-Wall and Ypsilanti lattices, Adv. Math. 196 (2005), no. 1, 147-192.

[14] R. L. Griess, Jr. and C. H. Lam, EE -lattices and dihedral groups, Pure Appl. Math. Q. 7 (2011), no. 3, 621-743.

[15] C. H. Lam, Automorphism group of an orbifold vertex operator algebra associated with the Leech lattice, in: Vertex Operator Algebras, Number Theory and Related Topics, 127-138, Contemp. Math. 753, Amer. Math. Soc., Providence, RI, 2020.

[16] _ Cyclic orbifolds of lattice vertex operator algebras having group-like fusions, Lett. Math. Phys. 110 (2020), no. 5, 1081-1112.

[17] Some observations about the automorphism groups of certain orbifold vertex operator algebras, to appear in RIMS Kôkyûroku Bessatsu.

[18] C. H. Lam and H. Shimakura, Extra automorphisms of cyclic orbifolds of lattice vertex operator algebras, arXiv:2103.08085.

[19] C. H. Lam and H. Yamauchi, On 3-transposition groups generated by $\sigma$-involutions associated to $c=4 / 5$ Virasoro vectors, J. Algebra 416 (2014), 84-121.

[20] J. Lepowsky, Calculus of twisted vertex operators, Proc. Nat. Acad. Sci. U.S.A. 82 (1985), no. 24, 8295-8299. 
[21] M. Miyamoto, $C_{2}$-cofiniteness of cyclic-orbifold models, Comm. Math. Phys. 335 (2015), no. 3, 1279-1286.

[22] H. Shimakura, The automorphism group of the vertex operator algebra $V_{L}^{+}$for an even lattice L without roots, J. Algebra 280 (2004), no. 1, 29-57.

[23] Math. Z. 252 (2006), no. 4, 849-862.

[24] Lifts of automorphisms of vertex operator algebras in simple current extensions, Math. Z. 256 (2007), no. 3, 491-508.

Hsian-Yang Chen

National University of Tainan, Tainan 70005, Taiwan

E-mail address: hychen@mail.nutn.edu.tw

Ching Hung Lam

Institute of Mathematics, Academia Sinica, Taipei 10617, Taiwan

E-mail address: chlam@math.sinica.edu.tw 